\title{
Robust pole placement for plants with semialgebraic parametric uncertainty
}

\author{
V. Cerone ${ }^{* \diamond}$, D. Piga ${ }^{\S}$, D. Regruto*
}

\begin{abstract}
In this paper we address the problem of robust pole placement for linear-time-invariant systems whose uncertain parameters are assumed to belong to a semialgebraic region. A dynamic controller is designed in order to constrain the coefficients of the closed-loop characteristic polynomial within prescribed intervals. Two main topics arising from the problem of robust pole placement are tackled by means of polynomial optimization. First, necessary conditions on the plant parameters for the existence of a robust controller are given. Then, the set of all admissible robust controllers is sought. Convex relaxation techniques based on sum-of-square decomposition of positive polynomials are used to efficiently solve the formulated optimization problems through semidefinite programming techniques.
\end{abstract}

Index Terms - Robust coprimeness, Robust pole placement, Semialgebraic uncertainty, Sum-of-square decomposition.

\section{INTRODUCTION}

Pole placement is a widely adopted technique to design feedback controllers for linear-time-invariant (LTI) systems (see, e.g., [1], [2]). Indeed, one of the most interesting properties of such a control design technique is the possibility to arbitrarily assign the roots of the closed-loop characteristic polynomial. In the case of plants described by proper transfer function, the well known Diophantine equation has to be solved (see, e.g., [3]). In the case of plants with parametric uncertainty, different techniques can be found in the literature to design robust controllers through the solution of uncertain Diophantine equation. More precisely, in [4] the set of admissible controllers is evaluated and, among them, the controller closest in some norm to a given nominal controller is chosen. In [5], the authors exploit linear programming (LP) optimization techniques for designing fixed-order controllers with guaranteed and robust performance. The designed controller is robust in itself, in the sense that the closed-loop system guarantees stability and desired performance also for perturbations on the controller parameters. Similar results are obtained in [6] by solving interval Diophantine equations through interval analysis techniques, and in [7], where a twodegree-of-freedom methodology for designing a controller with robust stability and performance is presented. All the papers mentioned above consider the case of plants with interval uncertainty on the parameters. The case of plants

* V. Cerone and D. Regruto are with the Dipartimento di Automatica e Informatica, Politecnico di Torino, corso Duca degli Abruzzi 24, 10129 Torino, Italy; e-mail: vito.cerone@polito.it, diego.regruto@polito.it; Tel: +39-011-090 7064; Fax: +39-0110907198

$\S$ D. Piga is with the Delft Center for Systems and Control, Delft University of Technology, Mekelweg 2, Delft, 2628 CD, The Netherlands; email: D.Piga@tudelft.nl

$\diamond$ Corresponding author with parameters belonging to a polytope is considered in [8] and [9], where a robust controller is designed in order to assign a closed-loop characteristic polynomial over a stability domain specified as a polytopic region on the space of characteristic polynomial coefficients. The same type of parameter uncertainty is assumed in [10] and [11], where semidefinite programming (SDP) optimization techniques are employed to construct a convex inner approximation of the stability domain in the characteristic polynomial coefficients space. Indeed, the assumption of either interval uncertainty or polytopic uncertainty on the plant parameters might introduce conservativeness in robust controller design. As a matter of fact, in the case of interval uncertainties, each plant parameter is constrained to vary within an uncertainty range independently on the other parameters, while, in the case of polytopic uncertainty description, only a linear dependance among plant parameters can be considered. Motivated by this fact, robust pole placement techniques are proposed in [12], [13], [14], [15] to deal with uncertain plants with parameters belonging to a convex ellipsoidal region. In this way, a quadratic dependence of parameter variations can be modeled. Besides, this kind of uncertainty description often arises from parameter identification procedures, where the identified parameters are guaranteed to belong to ellipsoidal regions with a certain level of probability [16].

In this paper, we address the problem of robust pole placement when the plant parameters are assumed to lie into nonconvex semialgebraic regions, which include, indeed, interval, polytopic and ellipsoidal uncertainty sets as special convex cases. To the author's best knowledge, no contribution can be found in the literature addressing this problem. It is worth remarking that a semialgebraic description of the parameter uncertainty description allows us to consider any kind of polynomial dependence among the plant parameters. For instance, as recently shown in [17], [18], [19], this type of parameter uncertainty description arises from bounded error-in-variable identification of LTI systems, that is identification of linear dynamical systems when both the input and the output measurements are corrupted by additive bounded noise.

The paper is organized as follows. In Section II the problem of robust control synthesis is formulated in terms of solution of a robust Diophantine equation, in order to guarantee that the coefficients of the closed-loop characteristic polynomial lie in desired intervals for all possible values of the plant parameters belonging to the considered semialgebraic uncertainty set. It is known that, for zero-uncertainty plants, a necessary condition so that there exists a solution to the Dio- 
phantine equation for any arbitrary characteristic polynomial is that numerator and denominator of the transfer function describing the plant are coprime [3]. By the same token, in the case of uncertain plants, numerator and denominator have to be coprime for all possible values of the parameters in the uncertainty region. A novel approach to evaluate robust coprimess of uncertain polynomials with coefficients belonging to a semialgebraic set is presented in Section III. In Section IV, convex relaxation techniques based on sumof-square (SOS) decomposition of positive polynomials are used to efficiently solve the robust Diophantine equation formulated in Section II. The reported simulated examples show the effectiveness of the proposed approach.

\section{Problem formulation}

Let us consider the feedback control system depicted in Fig. 1. The plant $\mathbf{G}$ to be controlled is a linear time invariant (LTI) system of order $n$ described by the strictly proper rational transfer function

$$
\mathbf{G}(s, \mathbf{p})=\frac{N_{p}(s, \mathbf{p})}{D_{p}(s, \mathbf{p})},
$$

where $N_{p}(s, \mathbf{p})$ and $D_{p}(s, \mathbf{p})$ are polynomials in the Laplace variable $s$, i.e.

$$
\begin{gathered}
N_{p}(s, \mathbf{p})=b_{n-1}(\mathbf{p}) s^{n-1}+b_{n-2}(\mathbf{p}) s^{n-2}+\cdots+b_{0}(\mathbf{p}), \\
D_{p}(s, \mathbf{p})=a_{n}(\mathbf{p}) s^{n}+a_{n-1}(\mathbf{p}) s^{n-1}+\cdots+a_{0}(\mathbf{p}) .
\end{gathered}
$$

Coefficients $\mathbf{a}(\mathbf{p})=\left[a_{n}(\mathbf{p}), a_{n-1}(\mathbf{p}), \cdots, a_{0}(\mathbf{p})\right]$ and $\mathbf{b}(\mathbf{p})=\left[b_{n-1}(\mathbf{p}), b_{n-2}(\mathbf{p}), \cdots, b_{0}(\mathbf{p})\right]$ depend polynomially on a set of parameters $\mathbf{p}=\left[p_{1}, p_{2}, \cdots, p_{n p}\right]$, which are assumed to belong to a bounded semialgebraic region $\mathcal{P} \subseteq \mathbb{R}^{n p}$ and $a_{n}(\mathbf{p})$ is such that $a_{n}(\mathbf{p}) \neq 0 \forall \mathbf{p} \in \mathcal{P}$.

Remark 1: Since parameters $\mathbf{p}$ belong to a bounded semialgebraic set and coefficients $\mathbf{a}$ and $\mathbf{b}$ depend polynomially on $\mathbf{p}$, then also $\mathbf{a}$ and $\mathbf{b}$ belong to a bounded semialgebraic set $\mathcal{C}$ defined as $\mathcal{C}:\left\{(\mathbf{a}, \mathbf{b}): g_{j}(\mathbf{a}, \mathbf{b}, \mathbf{p}) \geq 0, j=1, \ldots, n g\right\}$, where $g_{j}$, with $j=1, \ldots, n g$, are appropriate polynomials taking into account the constraints describing $\mathcal{P}$ and the dependance of coefficients $\mathbf{a}$ and $\mathbf{b}$ on parameters $\mathbf{p}$.

In this paper we address the problem of robust-pole placement, whose formulation is provided below.

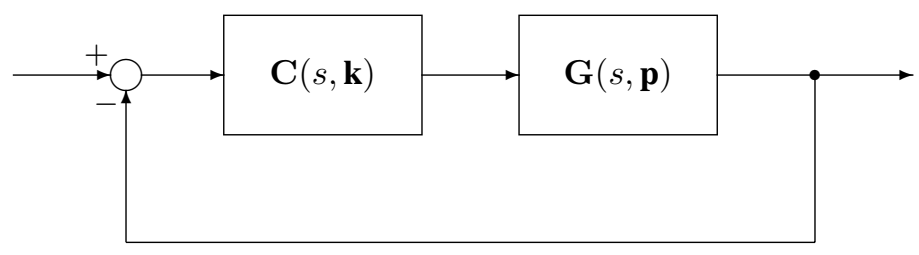

Fig. 1. Feedback control system
Problem 1: Robust pole placement

Design the parameters $\mathbf{k}=\left[k_{1}, k_{2}, \cdots, k_{2 r+2}\right]$ of an $r$-order dynamic controller $\mathbf{C}(s)$ :

$$
\mathbf{C}(s, \mathbf{k})=\frac{N_{c}(s, \mathbf{k})}{D_{c}(s, \mathbf{k})}=\frac{k_{1} s^{r}+k_{2} s^{r-1}+\cdots+k_{r}}{k_{r+1} s^{r}+k_{r+2} s^{r-1}+\cdots+k_{2 r+2}},
$$

in order to constrain each coefficient $f_{j}$, with $j=0, \ldots, r+$ $n$, of the uncertain closed-loop characteristic polynomial $F(s, \mathbf{p}, \mathbf{k})$ :

$$
\begin{aligned}
F(s, \mathbf{p}, \mathbf{k}) & =D_{c}(s, \mathbf{k}) D_{p}(s, \mathbf{p})+N_{c}(s, \mathbf{k}) N_{p}(s, \mathbf{p})= \\
& =f_{r+n} s^{n+r}+f_{r+n-1} s^{n+r-1}+\cdots+f_{0}
\end{aligned}
$$

to lie in a given interval $\left[\underline{f}_{j} ; \bar{f}_{j}\right]$ for all uncertain plants $\mathbf{G}(s, \mathbf{p})$ with $\mathbf{p} \in \mathcal{P}$.

In the case of plant models $\mathbf{G}$ without uncertainty, a solution of the Diophantine equation (5) exists for any arbitrary characteristic polynomial $F(s)$ if and only if $r \geq n-1$ and the plant $\mathbf{G}$ has no common zero-pole pairs. Therefore, a necessary condition which guarantees the existence of a solution to the robust pole placement problem is that polynomials $N_{p}(s, \mathbf{p})$ and $D_{p}(s, \mathbf{p})$ are coprime for all values of parameters $\mathbf{p} \in \mathcal{P}$, or equivalently, for all values of the coefficients $\mathbf{a}$ and $\mathbf{b}$ in $\mathcal{C}$. The following Lemma from commutative algebra provides a way to check robust coprimeness of two polynomials with uncertain coefficients.

Lemma 1: Let Sylvester matrix $S(\mathbf{a}, \mathbf{b}, \mathbf{p})$ associated with $N_{p}(s, \mathbf{p})$ and $D_{p}(s, \mathbf{p})$ be a $(2 n-1) \times(2 n-1)$ square matrix defined as

$S(\mathbf{a}, \mathbf{b}, \mathbf{p})=\left(\begin{array}{ccccccc}0 & \cdots & 0 & a_{n} & 0 & \cdots & 0 \\ b_{n-1} & \ddots & 0 & a_{n-1} & a_{n} & \ddots & 0 \\ \vdots & \ddots & 0 & \vdots & a_{n-1} & \ddots & 0 \\ \vdots & \ddots & \vdots & \vdots & \ddots & \ddots & \ddots \\ b_{0} & \ddots & b_{n-1} & a_{0} & \ddots & \ddots & a_{n-1} \\ 0 & \ddots & b_{n-2} & 0 & a_{0} & \ddots & a_{n-2} \\ \vdots & \ddots & \vdots & \vdots & \ddots & \ddots & \vdots \\ 0 & \cdots & b_{0} & 0 & \cdots & \cdots & a_{0}\end{array}\right)$

It is worth noting that the entries $a_{0}, \ldots, a_{n}$ and $b_{0}, \ldots, b_{n-1}$ of matrix $S(\mathbf{a}, \mathbf{b}, \mathbf{p})$ are functions of parameter $\mathbf{p}$. In the sequel, in order to simplify the notation, we will denote as $\tilde{S}$ the Sylvester matrix $S(\mathbf{a}, \mathbf{b}, \mathbf{p})$. The uncertain polynomials $N_{p}(s, \mathbf{p})$ and $D_{p}(s, \mathbf{p})$ are robustly coprime if and only if the Sylvester matrix $\tilde{S}$ associated with $N_{p}(s, \mathbf{p})$ and $D_{p}(s, \mathbf{p})$ is robustly nonsingular, that is, it is nonsingular for all values of the coefficients $\mathbf{a}$ and $\mathbf{b}$ in $\mathcal{C}$.

In the following section a SOS-based method to check robust nonsingularity of Sylvester matrixes with uncertain coefficients is presented. Then, in Section IV, we show how to design a controller to solve the robust pole placement formulated in Problem 1. 


\section{ON THE ROBUST NONSINGULARITY OF UNCERTAIN SYLVESTER MATRIXES}

Checking robust nonsingularity of uncertain matrixes is an NP-hard problem (see, e.g. [20], [21]) and it has been extensively treated in the literature in the case of interval matrixes (see [22], [23], [24]). Unfortunately, the approaches proposed in [22], [23], [24] to evaluate if an interval Sylvester matrix is robustly nonsingular provide conservative nonsingularity conditions since each occurrence of an interval coefficient in the Sylvester matrix has to be handled as if it was a different interval coefficient (see [6]). In this section we present a new approach to evaluate robust nonsingularity of uncertain Sylvester matrixes. The advantages of the presented method with respect to the approaches in [22], [23], [24] are mainly the following:

- uncertain coefficients appearing in the Sylvester matrix may belong to a semialgebraic set;

- the dependence among same coefficients in the Sylvester matrix is not lost. Therefore, in the case of Sylvester matrixes with interval polynomial coefficients, the approach proposed in the paper provides less conservative nonsingularity conditions.

The following theorem provides necessary and sufficient conditions to check robust nonsingularity of uncertain Sylvester matrixes.

Theorem 1: Let $\mathcal{S}(\mathbf{a}, \mathbf{b})$ be the set of uncertain Sylvester matrixes associated with polynomials $N_{p}(s, \mathbf{p})$ and $D_{p}(s, \mathbf{p})$ with coefficients $\mathbf{a}, \mathbf{b} \in \mathcal{C}$, i.e.

$$
\mathcal{S}(\mathbf{a}, \mathbf{b})=\{\tilde{S}: \mathbf{a}, \mathbf{b} \in \mathcal{C}\} .
$$

The Sylvester matrix $\tilde{S}$ in (6) is robustly nonsingular on $\mathcal{C}$ if and only if the solution of the following optimization problem is bounded:

$$
\begin{aligned}
& f^{*}=\max _{x \in \mathbb{R}^{2 n-1}}\|x\|_{2}^{2} \\
& \text { s.t. } \\
& \tilde{S} x=0 ; \quad \tilde{S} \in \mathcal{S}(\mathbf{a}, \mathbf{b}) .
\end{aligned}
$$

or equivalently if and only if the set

$$
\mathcal{F}=\left\{x \in \mathbb{R}^{2 n-1}: \tilde{S} x=0 ; \quad \tilde{S} \in \mathcal{S}(\mathbf{a}, \mathbf{b}) ; \quad\|x\|_{2}^{2} \geq \varepsilon\right\}
$$

is empty for any $\varepsilon \in \mathbb{R}: \varepsilon>0$.

Proof: We first prove the "only if " part. If matrix $\tilde{S}$ is robustly nonsingular, then, for all $\tilde{S} \in \mathcal{S}(\mathbf{a}, \mathbf{b})$, only the trivial solution $x=0$ satisfies the constraint $\tilde{S} x=0$. Therefore, the solution of problem (8) is equal to zero, thus bounded. The "if" part is proven by contradiction. Assume that $\tilde{S}$ is not robustly nonsingular. This means that there exists a value of $\mathbf{a}, \mathbf{b}$ in $\mathcal{C}$ such that the solution of the linear system $\tilde{S} x$ is not the trivial one, i.e. $\tilde{S} x^{*}=0$ for some $x^{*} \neq$ 0 . Therefore, for every $\alpha \in \mathbb{R}$, also $x=\alpha x^{*}$ satisfies the constraint $\tilde{S} x=0$ and thus it belongs to the set of feasibility of problem (8). Then, solution to problem (8) is unbounded, contradicting the hypothesis. The second part of the Theorem follows from the fact that, when $\tilde{S}$ is robustly nonsingular, $x=0$ is the only solution to equalities $\tilde{S} x=0$ for all $\tilde{S} \in \mathcal{S}(\mathbf{a}, \mathbf{b})$.

On the basis of Theorem 1, checking robust nonsingularity of the Sylvester matrix on $\mathcal{C}$ is recast into the nonconvex optimization problem (8). More precisely, (8) is a semialgebraic optimization problem since: (i) the objective function is quadratic; (ii) the set of constraints $\tilde{S} x=0$ involves the product between the variable $x$ and the uncertain coefficients $a$ and $b$; (iii) coefficients $a$ and $b$ lie in the semialgebraic set $\mathcal{C}$.

LMI-relaxation techniques based on Putinar's Positivstellensatz [25] have been proposed in order to relax nonconvex polynomial optimization problems into a hierarchy of convex SDP problems, whose solutions are proven to monotonically converge to the optimal value of the original nonconvex problems (see, e.g., [26], [27] and the survey paper [28] for an extensive review on this topic). Application of such LMI-based techniques to problem (8) leads to Theorem 2, whose statement is reported below. For a given integer $\delta$ such that $2 \delta \geq$ $\max \left\{2, \operatorname{deg}\left(g_{1}\right), \ldots, \operatorname{deg}\left(g_{n g}\right)\right\}$, let us consider the convex SDP problem

$$
\begin{aligned}
& f_{\delta}^{s o s}=\min _{\gamma \in \mathbb{R}} \gamma \\
& \text { s.t. } \\
& \gamma-\|x\|_{2}^{2}=\sigma_{0}+\sum_{j=1}^{n g} \sigma_{j} g_{j}(\mathbf{a}, \mathbf{b}, \mathbf{p})+\sum_{i=1}^{m} \lambda_{i} \tilde{S}_{i} x, \\
& \text { for some } \sigma_{0}, \sigma_{j} \in \Sigma[x, \mathbf{a}, \mathbf{b}, \mathbf{p}], \lambda_{i} \in \mathbb{R}[x, \mathbf{a}, \mathbf{b}, \mathbf{p}] \\
& \operatorname{deg}\left(\sigma_{0}\right), \operatorname{deg}\left(\sigma_{j} g_{j}\right), \operatorname{deg}\left(\lambda_{i} \tilde{S}_{i} x\right) \leq 2 \delta
\end{aligned}
$$

where $\tilde{S}_{i}$ is the $i$-th row of Sylvester matrix $\tilde{S} ; \mathbb{R}[x, \mathbf{a}, \mathbf{b}, \mathbf{p}]$ is the ring of polynomials in the variables $(x, \mathbf{a}, \mathbf{b}, \mathbf{p})$ and $\Sigma[x, \mathbf{a}, \mathbf{b}, \mathbf{p}]$ is the set of SOS polynomials in the same variables.

Theorem 2: If $f_{\delta}^{\text {sos }}$ is bounded for some $\delta$ s.t. $2 \delta \geq$ $\max \left\{2, \operatorname{deg}\left(g_{1}\right), \ldots, \operatorname{deg}\left(g_{n g}\right)\right\}$, then the matrix $\tilde{S}$ is nonsingular on $\mathcal{C}$.

Proof: Let us rewrite the polynomial optimization problem (8) as

$$
\begin{aligned}
& f^{*}=\min _{\gamma \in \mathbb{R}} \gamma \\
& \text { s.t. } \\
& \gamma-\|x\|_{2}^{2} \geq 0, \quad \forall x, \tilde{S} \text { s.t. } \tilde{S} x=0 ; \quad \tilde{S} \in \mathcal{S}(\mathbf{a}, \mathbf{b}) .
\end{aligned}
$$

Indeed, $\gamma-\|x\|_{2}^{2}=\sigma_{0}+\sum_{j=1}^{n g} \sigma_{j} g_{j}(\mathbf{a}, \mathbf{b}, \underset{\widetilde{S}}{\mathbf{p}})+\sum_{i=1}^{m} \lambda_{i} \tilde{S}_{i} x$ is nonnegative $\forall x, \tilde{S}$ s.t. $\tilde{S} x=0 ; \quad \tilde{S} \in \mathcal{S}(\mathbf{a}, \mathbf{b})$. In fact, when $\tilde{S} x=0$ and $\tilde{S} \in \mathcal{S}(\mathbf{a}, \mathbf{b}), \gamma-\|x\|_{2}^{2}=\sigma_{0}+$ $\sum_{j=1}^{n g} \sigma_{j} g_{j}(\mathbf{a}, \mathbf{b}, \mathbf{p})+\sum_{i=1}^{m} \lambda_{i} \tilde{S}_{i} x \geq 0$ since $\tilde{S}_{i} x=0$ $g_{j}(\mathbf{a}, \mathbf{b}, \mathbf{p}) \geq 0$ and $\sigma_{0}, \sigma_{j}$, with $j=1, \ldots, n g$, are sumof-square polynomials, hence nonnegative. Then, $f_{\delta}^{s o s}$ is an 
upper bound of $f^{*}$, i.e. $f_{\delta}^{s o s} \geq f^{*}$. Therefore, when $f_{\delta}^{s o s}$ is bounded, $f^{*}$ is bounded as well, and from Theorem 1, uncertain Sylvester matrix $\tilde{S}$ is robustly nonsingular on $\mathcal{C}$.

Remark 2: Alternatively, from the second part of Theorem 1 , robust nonsingularity of matrix $\tilde{S}$ on $\mathcal{C}$ can be checked by evaluating emptiness of the set $\mathcal{F}$. On the basis of Positivstellensatz, emptiness of a semialgebraic set can be efficiently evaluated through semidefinite programming. More precisely, $\mathcal{F}$ is empty if there exists a solution to the following SDP feasibility problem for some integer $\delta$ :

$$
\begin{aligned}
& \sigma_{0}+\sum_{j=1}^{n g} \sigma_{j} g_{j}(\mathbf{a}, \mathbf{b}, \mathbf{p})+\sigma_{n g+1}\left(\|x\|_{2}^{2}-\varepsilon\right)+\sum_{i=1}^{m} \lambda_{i} \tilde{S}_{i} x=0 \\
& \text { for some } \sigma_{0}, \sigma_{1}, \ldots, \sigma_{n g+1} \in \Sigma[x, \mathbf{a}, \mathbf{b}, \mathbf{p}] \\
& \lambda_{i} \in \mathbb{R}[x, \mathbf{a}, \mathbf{b}, \mathbf{p}] \\
& \operatorname{deg}\left(\sigma_{0}\right), \operatorname{deg}\left(\sigma_{j} g_{j}\right), \operatorname{deg}\left(\sigma_{n g+1}\|x\|_{2}^{2}\right), \operatorname{deg}\left(\lambda_{i} \tilde{S}_{i} x\right) \leq 2 \delta .
\end{aligned}
$$

Example 1: Let us consider the interval Sylvester matrix associated with the uncertain polynomials $N_{p}(s)=b_{2} s^{2}+b_{1} s+b_{0}$ and $D_{p}(s)=s^{3}+a_{2} s^{2}+a_{1} s+a_{0}$ with interval coefficients $b_{2} \in[3-\Delta ; 3+\Delta] ; b_{1} \in$ $[8-\Delta ; 8+\Delta] ; b_{0} \in[-5-\Delta ;-5+\Delta] ; a_{2} \in$ $[4-\Delta ; 4+\Delta] ; a_{1} \in[3-\Delta ; 3+\Delta] ; a_{0} \in[-6-\Delta ;-6+\Delta]$, where $\Delta \geq 0$ denotes the radius of the coefficient uncertainty intervals. In this example, different values $\Delta$ are considered. In particular, the largest value of $\Delta$ such that polynomials $N_{p}(s)$ and $D_{p}(s)$ are guaranteed to be robustly coprime is equal to 0.208 when the algorithm in [24] is used to evaluate robust nonsingularity of the associated interval Sylvester matrix, while it is equal to 0.201 when the method in [22] is used. On the other hand, by using results in Theorem 2, we get that polynomials $N_{p}(s)$ and $D_{p}(s)$ are guaranteed to be robustly coprime for $\Delta \leq 0.420$. It is worth remarking that, although Theorem 2 only provides a sufficient condition for checking robust nonsingularity of Sylvester matrixes, $\Delta=0.420$ is a good approximation of the exact radius of robust coprimeness of the considered polynomials, in fact, for instance, polynomials $N_{p}(s)$ and $D_{p}(s)$ with coefficients $b_{2}=3.4114, b_{1}=7.6088 b_{0}=-4.6509, a_{2}=4.4206$, $a_{1}=2.5728$ and $a_{0}=-5.5760$, belonging to uncertainty intervals with $\Delta=0.4273$, are not coprime since they have a common root equal to -2.7298 .

\section{Design OF ROBUST CONTROLLER}

In this section we show how to design the controller parameters $\mathbf{k}$ in order to solve the robust pole placement formulated in Problem 1. Let us rewrite the Diophantine equation in (5) in the matrix form:

$$
\tilde{S} \mathbf{k}=\mathbf{f},
$$

where $\mathbf{f}=\left[f_{n+r}, f_{n+r-1}, \ldots, f_{0}\right]$ is the vector of the coefficients of the closed-loop characteristic polynomial $\mathbf{F}(s, \mathbf{p}, \mathbf{k})$. The robust pole placement problem can be reformulated in terms of the following robust feasibility problem.

Problem 2: Find controller parameters $\mathbf{k} \in \mathbb{R}^{2 r+2}$ such that

$$
\left\{\begin{array}{l}
\tilde{S}_{i} \mathbf{k}-\tilde{f}_{i}>0 \\
\bar{f}_{i}-\tilde{S}_{i} \mathbf{k}>0
\end{array} \quad \forall \mathbf{p} \in \mathcal{P} ; \quad i=0, \ldots, n+r,\right.
$$

or equivalently

$$
\left\{\begin{array}{l}
\tilde{S}_{i} \mathbf{k}-\tilde{f}_{i}>0 \\
\bar{f}_{i}-\tilde{S}_{i} \mathbf{k}>0
\end{array} \quad \forall \mathbf{a}, \mathbf{b} \in \mathcal{C} ; \quad i=0, \ldots, n+r\right.
$$

Note that (15) is a robust polynomial feasibility problem because of the product between the controller parameters $\mathbf{k}$ and the uncertain plant coefficients $\mathbf{a}$ and $\mathbf{b}$, and because $\mathbf{a}$ and $\mathbf{b}$ belong to the semialgebraic region $\mathcal{C}$. By using Positivstellensatz, Problem 2 can be solved through SDP optimization, as shown instated by the following theorem.

Theorem 3: For a given integer $\delta$ such that $2 \delta \geq$ $\max \left\{2, \operatorname{deg}\left(g_{1}\right), \ldots, \operatorname{deg}\left(g_{n g}\right)\right\}$, let us consider the following SDP feasibility problem where $\mathbf{k} \in \mathbb{R}^{2 r+2}$ is the design parameter:

$$
\left\{\begin{array}{l}
\tilde{S}_{i} \mathbf{k}-\underline{f}_{i}=\sigma_{0, i}(\mathbf{a}, \mathbf{b}, \mathbf{p})+\sum_{j=1}^{n g} \sigma_{j, i}(\mathbf{a}, \mathbf{b}, \mathbf{p}) g_{j}(\mathbf{a}, \mathbf{b}, \mathbf{p})+\varepsilon \\
\bar{f}_{i}-\tilde{S}_{i} \mathbf{k}=\chi_{0, i}(\mathbf{a}, \mathbf{b}, \mathbf{p})+\sum_{j=1}^{n g} \chi_{j, i}(\mathbf{a}, \mathbf{b}, \mathbf{p}) g_{j}(\mathbf{a}, \mathbf{b}, \mathbf{p})+\varepsilon \\
\text { for some } \sigma_{0, i}, \sigma_{1, i}, \ldots, \sigma_{n g, i} \in \Sigma[\mathbf{k}, \mathbf{a}, \mathbf{b}, \mathbf{p}] \\
\text { for some } \chi_{0, h}, \chi_{1, h}, \ldots, \chi_{n g, h} \in \Sigma[\mathbf{k}, \mathbf{a}, \mathbf{b}, \mathbf{p}] \\
\text { for some } \varepsilon \in \mathbb{R}: \varepsilon>0 ; \\
\operatorname{deg}\left(\sigma_{0, i}\right), \operatorname{deg}\left(\sigma_{1, i} g_{j}\right), \ldots, \operatorname{deg}\left(\sigma_{n g, i} g_{n g}\right) \leq 2 \delta \\
\operatorname{deg}\left(\chi_{0, i}\right), \operatorname{deg}\left(\chi_{1, i} g_{j}\right), \ldots, \operatorname{deg}\left(\chi_{n g, i} g_{n g}\right) \leq 2 \delta \\
i=0, \ldots, r+n .
\end{array}\right.
$$

Let us denote with $\mathbf{k}_{\delta}^{*}$ a feasible solution for the convex SDP problem (16); then $\mathbf{k}_{\delta}^{*}$ belongs to set of admissible controller parameters given by (15).

Proof: Theorem 3 can be proven on the basis of similar reasonings used in the proof of Theorem 2. In fact, if polynomials $\widetilde{S}_{i} \mathbf{k}-\underline{f}_{i}$ and $\bar{f}_{i}-\tilde{S}_{i} \mathbf{k}$ can be written as

$$
\left\{\begin{array}{c}
\tilde{S}_{i} \mathbf{k}-\underline{f}_{i}=\sigma_{0, i}(\mathbf{a}, \mathbf{b}, \mathbf{p})+\sum_{j=1}^{n g} \sigma_{j, i}(\mathbf{a}, \mathbf{b}, \mathbf{p}) g_{j}(\mathbf{a}, \mathbf{b}, \mathbf{p})+\varepsilon \\
\bar{f}_{i}-\tilde{S}_{i} \mathbf{k}=\chi_{0, i}(\mathbf{a}, \mathbf{b}, \mathbf{p})+\sum_{j=1}^{n g} \chi_{j, i}(\mathbf{a}, \mathbf{b}, \mathbf{p}) g_{j}(\mathbf{a}, \mathbf{b}, \mathbf{p})+\varepsilon
\end{array}\right.
$$

for some SOS polynomials $\sigma_{j, i}$ and $\chi_{j, i}$, with $j=0, \ldots, n g$, and positive real number $\varepsilon$, then $\tilde{S}_{i} \mathbf{k}-\underline{f}_{i}$ and $\bar{f}_{i}-\tilde{S}_{i} \mathbf{k}$ are positive for all values of $\mathbf{a}$ and $\mathbf{b}$ belonging to $\mathcal{C}$. Therefore, if parameters $\mathbf{k}_{\delta}^{*}$ satisfy problem (16), then $\mathbf{k}_{\delta}^{*}$ is feasible for problem (15). 
Remark 3: Since $\mathcal{C}$ is a bounded set, on the basis of Putinar's Positivstellensatz, if polynomials $\tilde{S}_{i} \mathbf{k}-\underline{f}_{i}$ and $\bar{f}_{i}-\tilde{S}_{i} \mathbf{k}$ are positive on $\mathcal{C}$, then they can be always written as

$$
\left\{\begin{array}{l}
\tilde{S}_{i} \mathbf{k}-\underline{f}_{i}=\sigma_{0, i}(\mathbf{a}, \mathbf{b}, \mathbf{p})+\sum_{j=1}^{n g} \sigma_{j, i}(\mathbf{a}, \mathbf{b}, \mathbf{p}) g_{j}(\mathbf{a}, \mathbf{b}, \mathbf{p})+\varepsilon \\
\bar{f}_{i}-\tilde{S}_{i} \mathbf{k}=\chi_{0, i}(\mathbf{a}, \mathbf{b}, \mathbf{p})+\sum_{j=1}^{n g} \chi_{j, i}(\mathbf{a}, \mathbf{b}, \mathbf{p}) g_{j}(\mathbf{a}, \mathbf{b}, \mathbf{p})+\varepsilon \\
\text { for some } \sigma_{0, i}, \sigma_{1, i}, \ldots, \sigma_{n g, i} \in \Sigma[\mathbf{k}, \mathbf{a}, \mathbf{b}, \mathbf{p}] \\
\text { for some } \chi_{0, i}, \chi_{1, i}, \ldots, \chi_{n g, i} \in \Sigma[\mathbf{k}, \mathbf{a}, \mathbf{b}, \mathbf{p}] \\
\text { for some } \varepsilon \in \mathbb{R}: \varepsilon>0 .
\end{array}\right.
$$

This means that, whenever a solution of problem (15) exists, then the convex SDP problem (16) is guaranteed to be feasible for $\delta$ large enough. Nevertheless, in practice, whenever problem (15) is feasible, then the SDP problem (16) is feasible also for small values of $\delta$.

Example 2: Let us consider the second-order plant with transfer function

$$
\mathbf{G}(s, p)=\frac{N_{p}(s, \mathbf{p})}{D_{p}(s, \mathbf{p})}=\frac{p_{1} s+p_{2}}{s^{2}+p_{3} p_{4} s+p_{4}},
$$

where the uncertain parameters $p_{1}$ and $p_{2}$ belong to the semialgebraic region $\mathcal{P}_{12}$ :

$$
\begin{aligned}
\mathcal{P}_{12}= & \left\{\left(p_{1}, p_{2}\right) \in \mathbb{R}^{2}:\left(p_{1}-1.8\right)^{2}+\left(p_{2}-10\right)^{2} \leq 0.25 ;\right. \\
& p_{2}\left[1+4\left(p_{1}-1.8\right)\right] \leq \frac{3}{4}-\frac{39}{4}\left[1+4\left(p_{1}-1.8\right)\right], \\
& \left.p_{2}\left[1+4\left(p_{1}-1.8\right)\right] \geq-\frac{3}{4}-\frac{39}{4}\left[1+4\left(p_{1}-1.8\right)\right]\right\} .
\end{aligned}
$$

while parameters $p_{3}$ and $p_{4}$ lie in the semialgebraic set $\mathcal{P}_{34}$ defined as:

$$
\begin{aligned}
\mathcal{P}_{34}= & \left\{\left(p_{3}, p_{4}\right) \in \mathbb{R}^{2}: p_{3} \geq-9.8 ; \quad p_{4} \leq 0.4 ;\right. \\
& \left.p_{4} \geq \frac{4}{5}\left(p_{3}+9.3\right)^{3}+0.3\right\} .
\end{aligned}
$$

Uncertainty sets $\mathcal{P}_{12}$ and $\mathcal{P}_{34}$ are shown, respectively, in Fig. 2 and in Fig. 3. Note that all systems belonging to the considered family of uncertain plants are not stable, since the coefficient $p_{3} p_{4}$ of the second term of the denominator $D_{p}(s, \mathbf{p})$ is negative for all pair of parameters $\left(p_{3}, p_{4}\right) \in \mathcal{P}_{34}$. First, robust coprimeness of the uncertain polynomials $N_{p}(s, \mathbf{p})$ and $D_{p}(s, \mathbf{p})$ describing the plant is checked through the algorithm presented in Section III. Then, a feedback controller is designed in order to obtain the closedloop characteristic polynomial:

$$
\mathbf{F}(s)=s^{3}+f_{2} s^{2}+f_{1} s+f_{0},
$$

with coefficients in the open intervals: $f_{2} \in(6 ; 12), f_{1} \in$ $(19 ; 35)$ and $f_{0} \in(25 ; 29)$. The spectral set of the family of desired characteristic polynomials is shown in Fig. 4.

The set of admissible controllers is obtained by solving the

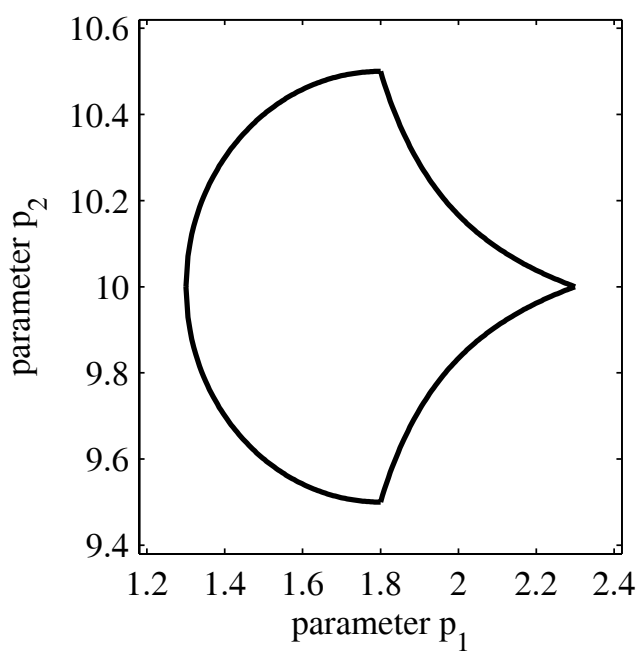

Fig. 2. Uncertainty set $\mathcal{P}_{12}$ (region inside black line) on parameters $p_{1}$ and $p_{2}$.

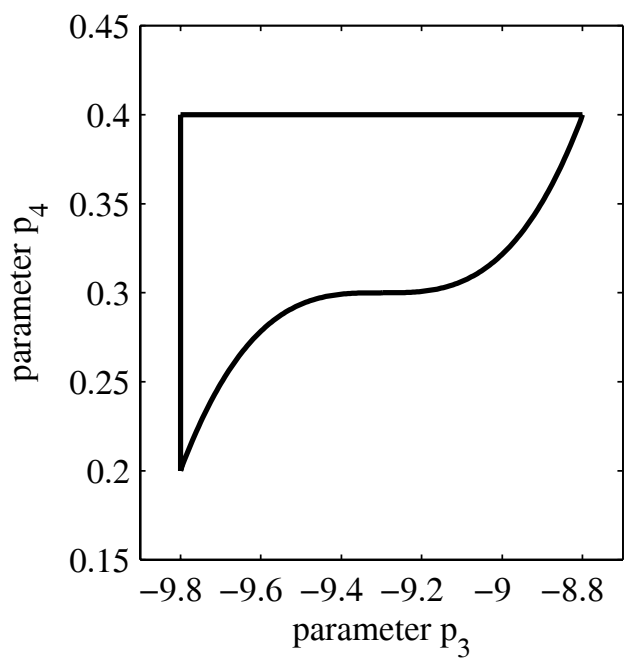

Fig. 3. Uncertainty set $\mathcal{P}_{34}$ (region inside black line) on parameters $p_{3}$ and $p_{4}$.

convex SDP problem (16) for $\delta=3$. For instance, a feasible controller $\mathbf{C}(s)$ satisfying the assigned specifications is given by:

$$
\mathbf{C}(s)=\frac{3.684 s+2.544}{s+5.186} .
$$

\section{CONCLUding REMARKS}

Synthesis of feedback robust controllers for linear-timeinvariant systems with uncertain parameters belonging to semialgebraic regions is addressed in the paper. Uncertain Diophantine equation is formulated in order to constrain the coefficients of the closed-loop characteristic polynomial to lie within given intervals. Two main topics related to Diophantine equation are discussed. First, a new approach to evaluate robust nonsingularity of the uncertain Sylvester matrix associated with the numerator and denominator of 


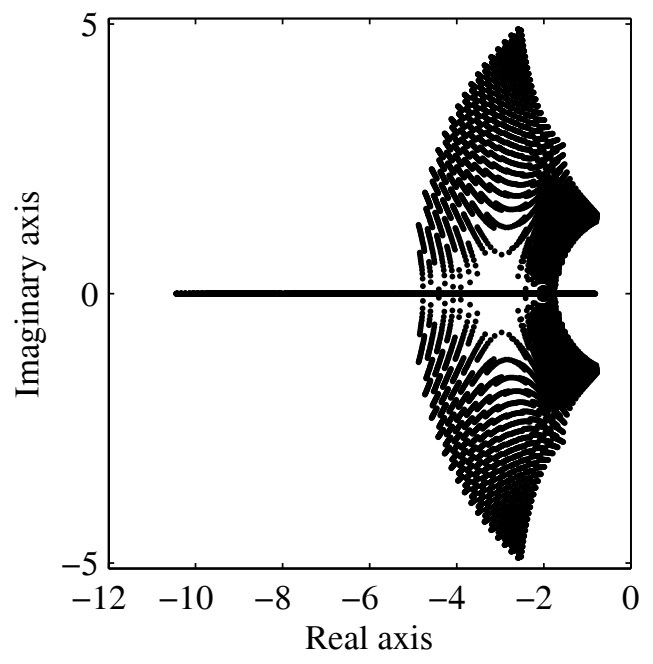

Fig. 4. Spectral set of the family of desired characteristic polynomials.

the transfer function describing the plant is presented. The problem of robust nonsingularity of the Silvester matrix is formulated as a nonconvex polynomial optimization problem, whose approximate solution is efficiently computed by means of sum-of-square convex-relaxation techniques. The discussed approach is able to deal with matrixes with uncertain entries belonging to a semialgebraic region and, in the case of interval uncertainty in the coefficients of the Sylvester matrix, the presented method provides less conservative conditions on the nonsingularity of Sylvester matrix with respect to previously published results. Finally, the design of a robust controller is reduced to a robust polynomial feasibility problem, which is efficiently solved through sum-of-square decomposition techniques similar to the ones exploited to check robust coprimeness of uncertain Sylvester matrixes. The reported simulated examples show the effectiveness of the presented results.

\section{ACKNOWLEDGments}

This research was developed while Dr. D. Piga was Ph.D student at the Politecnico di Torino.

\section{REFERENCES}

[1] W. Wonham, "On pole assignment in multi-input controllable linear systems," IEEE Transactions on Automatic Control, vol. 12, no. 6, pp. 660-665, 1967.

[2] M. Ait Rami, S. El Faiz, A. Benzaouia, and F. Tadeo, "Robust exact pole placement via an LMI-based algorithm," IEEE Transactions on Automatic Control, vol. 54, no. 2, pp. 394-398, 2009.

[3] C.-T. Chen, Linear System Theory and Design (3rd Ed.). New York: Oxford University Press, 1999.

[4] Y. C. Soh, R. J. Evans, I. R. Petersen, and R. E. Betz, "Robust pole assignment," Automatica, vol. 23, pp. 601-610, 1987.

[5] L. H. Keel and S. P. Bhattacharyya, "Robust stability and performance with fixed-order controllers," Automatica, vol. 35, pp. 1717-1724, 1999.

[6] A. D. S. Lordelo, E. A. Juzzo, and P. A. V. Ferreira, "Analysis and design of robust controllers using interval diophantine equation," Reliable Computing, vol. 12, no. 5, pp. 371-388, 2006.
[7] B. M. Patre and P. J. Deore, "Robust stability and performance for interval process plants," ISA Transactions, vol. 46, no. 3, p. 343349 , 2007.

[8] L. Jetto, "Strong stabilization over polytopes," IEEE Transactions on Automatic Control, vol. 44, pp. 1211-1216, 1999.

[9] U. Nurges, "Robust pole assignment via reflection coefficients of polynomials," Automatica, vol. 42, pp. 1223-1230, 2006.

[10] D. Henrion, M. Šebek, and V. Kučera, "Positive polynomials and robust stabilization with fixed-order controllers," IEEE Transactions on Automatic Control, vol. 48, no. 7, pp. 1178-1186, 2003.

[11] M. Ait Rami and D. Henrion, "A hierarchy of LMI inner approximations of the set of stable polynomials," Automatica, vol. 47, pp. $1455-1460,2011$

[12] O. D. Crisalle and R. K. Ballamudi, "Robust pole-placement technique for plants with ellipsoidally uncertain parameters," Chemical Enyineering Science, vol. 51, no. 12, pp. 3193 -3202, 1996.

[13] R. D. Braatz and O. D. Crisalle, "Robustness analysis for systems with ellipsoidal uncertainty," Int. J. Robust Nonlinear Control, vol. 8, pp. 1113-1117, 1998

[14] D. Henrion, M. Šebek, and V. Kučera, "LMIs for robust stabilization of systems with ellipsoidal uncertainty," IEEE Transactions on Automatic Control, vol. 48, no. 7, pp. 1178-1186, 2003.

[15] A. Sadeghzadeh, "Identification and robust control for systems with ellipsoidal parametric uncertainty by convex optimization," Asian Journal of Control, 2011. [Online]. Available: http://dx.doi.org/10.1002/asjc.437

[16] L. Ljung, System Identification, Theory for the User. Upper Saddle River: Prentince Hall, 1999.

[17] V. Cerone, D. Piga, and D. Regruto, "Improved parameters bounds for set-membership EIV problems," International Journal of Adaptive Control and Signal Processing, vol. 25, no. 3, pp. 208-227, 2011.

[18] — , "Enforcing stability constraints in set-membership identification of linear dynamic systems," Automatica, vol. 47, no. 11, pp. 24882494, 2011.

[19] — - "Set-membership error-in-variables identification through convex relaxation techniques," IEEE Transactions on Automatic Control, vol. 57, pp. 517-522, 2012.

[20] S. Polijak and J. Rohn, "Checking robust non-singularity is np-hard," Mathematics of Control, Signals, and Systems, vol. 6, no. 2, pp. 1-9, 1993.

[21] A. Nemirovskii, "Several NP-hard problems arising in robust stability analysis," Mathematics of Control, Signals, and Systems, vol. 6, no. 2 , pp. 99-105, 1993.

[22] H. Beeck, "Zur problematik der hullenbestimmung von intervallgeichungssystemen," in Interval Mathematics, Lecture Notes in Computer Science 29. Springer-Verlag, Berlin, 1975, pp. 150-159.

[23] G. Rex and J. Rohn, "Sufficient conditions for regularity and singularity of interval matrices," SIAM Journal on Mathematical Analysis and Applications, vol. 20, no. 2, p. 437445, 1998.

[24] C. Jansson and J. Rohn, "An algorithm for checking regularity of interval matrices," SIAM Journal on Mathematical Analysis and Applications, vol. 20, no. 3, pp. 756-776, 1999.

[25] M. Putinar, "Positive polynomials on compact semi-algebraic sets,' Indiana University Mathematics Journal, vol. 42, p. 969984, 1993.

[26] J. B. Lasserre, "Global optimization with polynomials and the problem of moments," SIAM Journal on Optimization, vol. 11, pp. 796-817, 2001.

[27] P. Parrillo, "Semidefinite programming relaxations for semialgebraic problems," Mathematical Programming, vol. 96, pp. 293-320, 2003.

[28] M. Laurent, "Sums of squares, moment matrices and optimization over polynomials," Emerging Applications of Algebraic Geometry, Vol. 149 of IMA Volumes in Mathematics and its Applications, M. Putinar and S. Sullivant (eds.), pp. 157-270, 2009. 